\title{
Prevalence and risk factors for extended-spectrum $\beta$-lactamase or AmpC-producing Escherichia coli in organic dairy herds in the Netherlands
}

\author{
I. M. G. A. Santman-Berends, ${ }^{, 1,2}$ M. A. Gonggrijp, ${ }^{* 1}$ J. J. Hage, ${ }^{*}$ A. E. Heuvelink, ${ }^{*}$ A. Velthuis, ${ }^{*}$ \\ T. J. G. M. Lam, ${ }^{*} \dagger$ and G. van Schaik ${ }^{*} \dagger$ \\ ${ }^{*}$ GD Animal Health, PO Box 9, 7400 AA Deventer, the Netherlands \\ †Department of Farm Animal Health, Faculty of Veterinary Medicine, Utrecht University, PO Box 80151, 3508 TD Utrecht, the Netherlands
}

\begin{abstract}
Extended-spectrum $\beta$-lactamase and AmpC-producing Escherichia coli (ESBL/AmpC) are an emerging problem and are hypothesized to be associated with antimicrobial use (AMU), and more specifically with the use of third- and fourth-generation cephalosporins. Whether ESBL/AmpC also occur in organic dairy herds, which have restricted AMU, is not known. Additionally, it is unknown whether, in addition to restricted AMU, other factors in organic herd management are associated with ESBL/AmpC herd status. The aim of this study was to estimate the prevalence of ESBL/ AmpC in organic dairy herds in the Netherlands. Subsequently, the relationships between the ESBL/AmpC herd status and AMU and between ESBL/AmpC herd status and farmers' management were assessed in organic dairy herds. For this study, 90 randomly selected, officially registered organic dairy herds were included. The ESBL/AmpC herd status was determined based on the bacteriological culture result of a slurry sample. The sensitivity of testing slurry samples for ESBL/ AmpC herd status is less than $100 \%$ for detecting herds with a low ESBL/AmpC prevalence. For that reason, herds that tested positive for ESBL/AmpC in slurry were defined as positive and herds with negative slurry samples were defined as unsuspected. A comprehensive questionnaire on management practices was conducted and records on specified antimicrobials that were provided to these herds by the veterinary service providers were obtained. From the data on antimicrobial supplies by the veterinarian, the animal daily defined dose of antimicrobials per farm per year $\left(\mathrm{DDDA}_{\mathrm{F}}\right)$ was calculated. Descriptive statistics were used to describe the relation between the ESBL/AmpC herd status
\end{abstract}

Received August 6, 2016.

Accepted October 7, 2016.

${ }^{1}$ Both authors contributed equally to this paper.

${ }^{2}$ Corresponding author: i.santman@gdanimalhealth.com and $\mathrm{DDDA}_{\mathrm{F}}$. Multivariable logistic regression models were used to evaluate management factors associated with the ESBL/AmpC herd status. We found ESBL/ AmpC in 12 of the 90 (13\%; 95\% confidence interval = $7-22 \%$ ) slurry samples from organic dairy herds. The median $\mathrm{DDDA}_{\mathrm{F}}$ in organic dairy herds was 0.5 , which was not significantly different between ESBL/AmpCpositive and unsuspected dairy herds. No association could be found between the use of different types of antimicrobials, such as third- and fourth-generation cephalosporins, and ESBL/AmpC herd status. Factors that were associated with higher odds of being ESBL/AmpC-positive were pig farms located within a 2 -km radius of the barn, applying parental treatment for clinical mastitis, and providing milk replacer to the female calves after colostrum intake. The prevalence of ESBL/AmpC in organic dairy herds appeared lower than the prevalence in previous studies conducted in conventional dairy herds. Apparently, ESBL/AmpC are also present in herds with low AMU; this indicates that other factors than AMU are also associated with ESBL/AmpC herd status.

Key words: dairy cattle, organic farming, antimicrobial use, extended-spectrum $\beta$-lactamase, antimicrobial resistance

\section{INTRODUCTION}

Antimicrobial resistance is an important problem in both veterinary and human medicine (Marshall and Levy, 2011). In Dutch livestock, antimicrobial resistance is mainly associated with Escherichia coli, Campylobacter, and Salmonella bacteria, and resistance in dairy cattle is considered to be low (MARAN, 2015). In recent years, many studies described the emergence of E. coli producing extended-spectrum $\beta$-lactamase (ESBL) and AmpC type $\beta$-lactamases (AmpC; Teale et al., 2005; Reist et al., 2013; Stefani et al., 2014). These ESBL/AmpC-producing E. coli (ESBL/AmpC) have been demonstrated to be related to resistance against 
third- and fourth-generation cephalosporins in humans and animals (Li et al., 2007; Snow et al., 2012; Gonggrijp et al., 2016), which are defined as critically important antimicrobials by the World Health Organization (www.who.int).

High prevalence of ESBL-producing E. coli in animals have been demonstrated in many studies and vary between countries and animal species (Wu et al., 2013; Valentin et al., 2014). In cattle, studies focused on ESBL/AmpC in veal, dairy, and beef cattle and showed prevalence ranging between 1 to $32.8 \%$ on an animal level and 35.4 to $86.7 \%$ on a herd level (Snow et al., 2012; Hordijk et al., 2013a; Schmid et al., 2013; Wu et al., 2013; Carmo et al., 2014; Dorado-García et al., 2016; Gonggrijp et al., 2016); these studies, however, included only conventionally farmed cattle. Although, hospital submission, travel, and human-human contact are considered important contamination routes for humans, pets and livestock are considered relevant sources for the colonization of humans with ESBL (Meyer et al., 2012; Wu et al., 2013; von Wintersdorff et al., 2014; Sharp et al., 2014).

In European registered organic dairy herds, a restricted antimicrobial usage (AMU) policy is applied that prohibits AMU unless it is prescribed by a veterinarian and with a maximum frequency of 3 treatments per cow per year (European Union regulation $834 / 2007$ and 889/2008; EC, 2007, 2008). Because of these restrictions, the use of third- and fourth-generation cephalosporins in organic herds generally is very limited. As the use of this type of antibiotic is known to be associated with the ESBL/AmpC herd status, it is hypothesized that the ESBL/AmpC herd prevalence in organic dairy herds is lower than in conventional dairy herds. Currently, no information exists that confirms or rejects this hypothesis. To our knowledge, only one study has evaluated ESBL/AmpC in an organic herd. Dolejska et al. (2011) found none of the 154 fecal samples (prevalence $<1 \%$ ) from a single organic dairy herd to test positive for ESBL/AmpC compared with $39 \%$ of the cattle in a single conventional dairy herd in the Czech Republic. Those authors suggested an association between AMU, especially cephalosporin use, and ESBL/AmpC herd status. Whether ESBL/AmpC occurs in organic dairy herds and to what extent is unknown. In addition, the relationship between ESBL/ AmpC herd status in organic dairy herds and AMU, and whether other factors are associated with ESBL/ AmpC herd status, is unknown.

The aim of the current study was to estimate the prevalence of ESBL/AmpC in organic dairy herds in the Netherlands. In addition, the relationships between the ESBL/AmpC herd status and AMU and the ESBL/
AmpC herd status and farmers' management in organic dairy herds were evaluated.

\section{MATERIALS AND METHODS}

\section{Definition of ESBL/AmpC Herd Status}

In our study, slurry sample levels were evaluated instead of individual feces samples to assign an ESBL/ AmpC herd status in accordance to Gonggrijp et al. (2016). The slurry samples could either contain fresh manure gathered from the scraper or, if no scraper was present, from 5 different places on the slatted floor. Because the slurry may not be sensitive enough to detect a low ESBL/AmpC prevalence within the herd, herds with ESBL/AmpC-negative slurry samples were described as unsuspected herds. Herds that tested positive for ESBL/AmpC in the slurry sample were described as positive herds.

\section{Study Population}

For this cross-sectional study, all 400 Dutch organic dairy farms that are officially registered in the SKAL database (control organization for organic farming, Zwolle, the Netherlands) were put in a random sequence and were contacted in that order by phone. All milking cows of the first 90 organic farmers that agreed to participate were included in the study and are described as herds in the remainder of this paper. These herds represent organic herds and have a low AMU compared with conventional herds. According to European Union regulation (EC, 2007, 2008), these herds are obliged to apply a very restrictive AMU policy.

Based on calculations in Win-Episcope 2.0 (Thrusfield et al., 2001), we estimated that with this population size it was possible to obtain a precise estimate (accepted error of $6 \%$ ) of the herd prevalence of ESBL/ AmpC in organic dairy herds (assuming 95\% confidence, $80 \%$ power, and an expected herd prevalence of $<10 \%$ ). In addition, we estimated that this population size enabled the possibility to detect risk factors for an ESBL/AmpC-positive herd status with an odds ratio of 3.5 or higher (assuming 95\% confidence, $80 \%$ power, and an expected prevalence of the risk factors of control herds of 20\%).

\section{Sampling and Laboratory Analyses}

All herds were sampled between September 1 and December 31, 2011. During the visits, a transport swab with Amies medium (Beldico, Duiven, the Netherlands) was used to swab the scraper at different places. When 
no scraper was present, slurry samples from 5 randomly selected places on the slatted floor were thoroughly mixed and sampled by using the transport swab. The slurry samples were cooled and transported to GD Animal Health (Deventer, the Netherlands) for laboratory analysis.

At the laboratory, the fecal sample was streaked onto MacConkey agar (Becton Dickinson, Franklin Lakes, NJ) supplemented with $1 \mathrm{mg} / \mathrm{L}$ of cefotaxime and then the swab was transferred into $10 \mathrm{~mL}$ of Luria-Bertani broth (Becton Dickinson) supplemented with $1 \mathrm{mg} / \mathrm{L}$ of cefotaxime (Sigma-Aldrich, St. Louis, MO). Both the plates and the broths were incubated aerobically overnight at $37^{\circ} \mathrm{C}$. After overnight incubation of the plates at $37^{\circ} \mathrm{C}$, presumptive $E$. coli colonies were confirmed as such using matrix-assisted laser desorption/ionization time-of-flight MS (Maldi Biotyper, Bruker, Billerica, MA). The Luria-Bertani broth and cefotaxime cultures were streaked onto MacConkey agar supplemented with cefotaxime and, following overnight incubation at $37^{\circ} \mathrm{C}$, typical E. coli colonies were confirmed as described above.

Confirmed E. coli isolates were examined for ESBL or AmpC production by the combination disc diffusion test using cefotaxime and ceftazidime with and without clavulanic acid (Becton Dickinson), according to CLSI guidelines (CLSI 2011), and cefoxitin (Dierikx et al., 2012), respectively. A more detailed description of the laboratory procedures used to isolate ESBL and AmpC can be found in Gonggrijp et al. (2016).

\section{Available Data on the Use of Antimicrobials}

All 90 participating farmers were asked permission to use the invoices that they received from their veterinary practitioners in 2011. These invoices were provided by either the farmer or the veterinary practice. Data from the invoices were digitalized based on the registration number (EAN-code) of the product, the product name, and the amount of antimicrobial that was supplied to each of the study herds (one supply was defined as one delivery on a certain date; SDA, 2014). In case antimicrobials were supplied that were not officially registered for use in cattle, we verified with the farmer whether these antimicrobials were used for cattle or for other species. If antimicrobials were used for other species, or if the species of the treated animal was unclear, the antimicrobials were removed from the data. Negative supplies of antimicrobials, representing corrections of delivered antimicrobials, were only included when the related delivery also took place during the study period. It was assumed that antimicrobials that were delivered by the veterinarian during 2011 were used in the same year (i.e., 2011). Based on the data on supplied antimicrobials, combined with data on herd size, age of the cattle that were present, and pharmaceutical information, the animal-defined daily dose of AMU per farm per year $\left(\mathbf{D D D A}_{\mathbf{F}}\right)$ was calculated as described by Gonggrijp et al. (2016). In short, for each of the herds, the total amount of treatable cattle weight in 2011 was calculated by multiplying the amount of delivered product with the registered amount of treatable weight per dose (milliliter, milligram, gram, piece, and so on) of the delivered product. Subsequently, the $\mathrm{DDDA}_{\mathrm{F}}$ was calculated by dividing the amount of the total treatable weight by the total weight of cattle present per herd in 2011.

In addition to the total $\mathrm{DDDA}_{\mathrm{F}}$ in 2011, the $\mathrm{DDDA}_{\mathrm{F}}$ for 3 separate application methods, AMU applied orally $\left(\mathrm{DDDA}_{\mathrm{F}, \text { oral }}\right), \mathrm{AMU}$ for dry cow treatment $\left(\mathrm{DDDA}_{\mathrm{F}, \mathrm{dry}}\right)$, and AMU for intramammary mastitis treatment other than dry cow treatment $\left(D_{D D A} A_{F, m a s t}\right)$, were calculated. The $\mathrm{DDDA}_{\mathrm{F}}$ per application method was calculated similar to the total $\mathrm{DDDA}_{\mathrm{F}}$, with only certain groups of antimicrobials and age categories involved. The same was done for the $\mathrm{DDDA}_{\mathrm{F}}$ per type of antimicrobial, specifically first- and second-generation cephalosporins, third- and fourth-generation cephalosporins, penicillins, tetracyclines, sulfonamides, combination antimicrobials, and other antimicrobials not previously mentioned.

\section{Questionnaire Data}

In the period between December 2011 and February 2012, all participating farmers were contacted by phone and a questionnaire was administered to obtain information on management factors such as housing facilities, hygiene, feeding regimen, milking procedures, udder health, and treatment regimens. The items included in the questionnaire are summarized by category of management practices (Table 1).

The study herds were randomly divided between 2 instructed technicians that conducted the questionnaires. The technicians that conducted the questionnaire were blind for the ESBL/AmpC herd status of the interviewed herds. The questions focused on 2011, which was the year in which the slurry samples were collected to evaluate the ESBL/AmpC herd status.

\section{Analyses}

The diagnostic results of the slurry samples were combined with the data on AMU and the results of the questionnaire. Stata version 13 (StataCorp, 2014) was used for analysis. Descriptive statistics on general herd characteristics, the DDDA , and the ESBL/ AmpC prevalence were calculated using frequency and 
summarizing tables. In addition, a potential effect of sampling was assessed by stratifying the ESBL/AmpC herd prevalence to the scraper versus the slatted floors sampling method.

A logistic regression model was used to evaluate the association between the ESBL/AmpC herd status and the DDDAF. The ESBL/AmpC herd status was included as a dependent variable and total $\mathrm{DDDA}_{\mathrm{F}}$, $\mathrm{DDDA}_{\mathrm{F}}$ per application method, or DDDAF per type of antibiotic as an independent variable.

To evaluate the possible associations between management factors and ESBL/AmpC herd status, a logistic regression model with a logit link function was used. In that model, the ESBL/AmpC herd status was included as dependent variable and management factors were included as independent variables. All management factors that were considered to be potentially associated with the ESBL/AmpC herd status, the sampling method (scraper versus slatted floor), and the information available through the questionnaire were included in the model after being screened in a univariable analysis. Risk factors with an association with the ESBL/AmpC herd status at a $P$-value of $<0.25$ entered the multivariable analysis. The multivariable analysis was conducted using a forward selection and elimination procedure. After each run, the variable with the lowest $P$-value in the univariable analysis entered the model until a final model was defined in which all variables had a $P \leq 0.05$ and had the lowest Akaike information criterion value (Akaike, 1974). Confounding was monitored by the change in the coefficient of a variable after removing another variable. If the change of the estimates exceeded $25 \%$ or 0.1 when the value of the estimate was between -0.4 and 0.4 , the variable was considered to be a confounder and was re-entered in the model. The robustness of the final model was checked by carrying out the same procedure with backward selection, in which at each run the variable with the highest $P$-value was removed. The model was considered robust when the forward and backward selection and elimination method resulted in the same final model. The coefficient of determination $\left(\mathrm{R}^{2}\right)$ was calculated to measure the proportion of variance explained by the final model.

\section{RESULTS}

\section{Descriptive Results and Prevalence of ESBL/AmpC}

The organic herds in our study housed a median of $51($ minimum $=9 ;$ maximum $=185)$ dairy cows $(>2 \mathrm{yr}$ old) and had a median milk 305-d production of 6,461 $\mathrm{kg}$ in the study period. The study herds were located throughout the Netherlands.

Table 1. Management factors that were potentially associated with extended-spectrum $\beta$-lactamase and AmpC-producing Escherichia coli (ESBL/AmpC) and were evaluated in a questionnaire in organic dairy herds

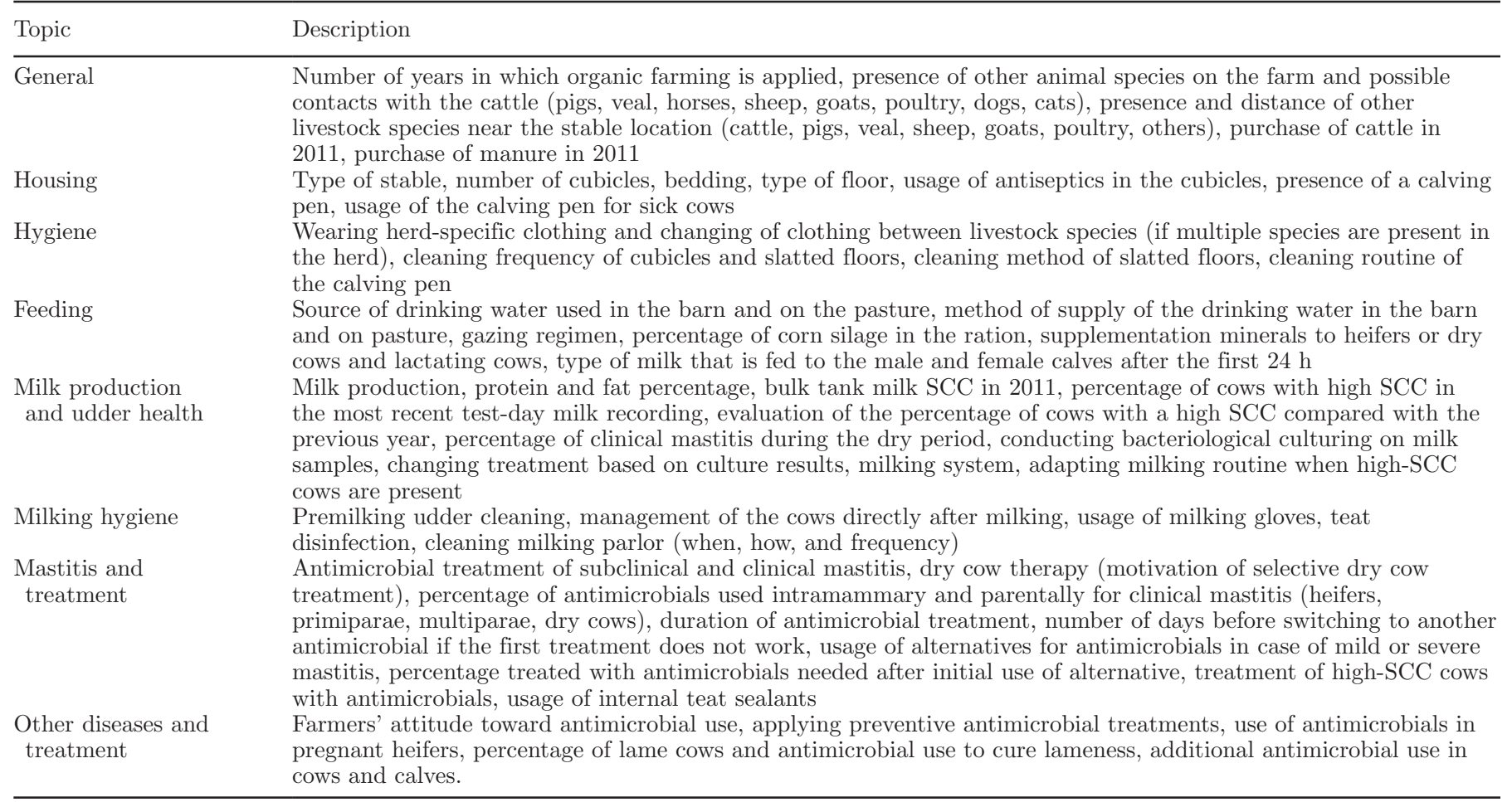




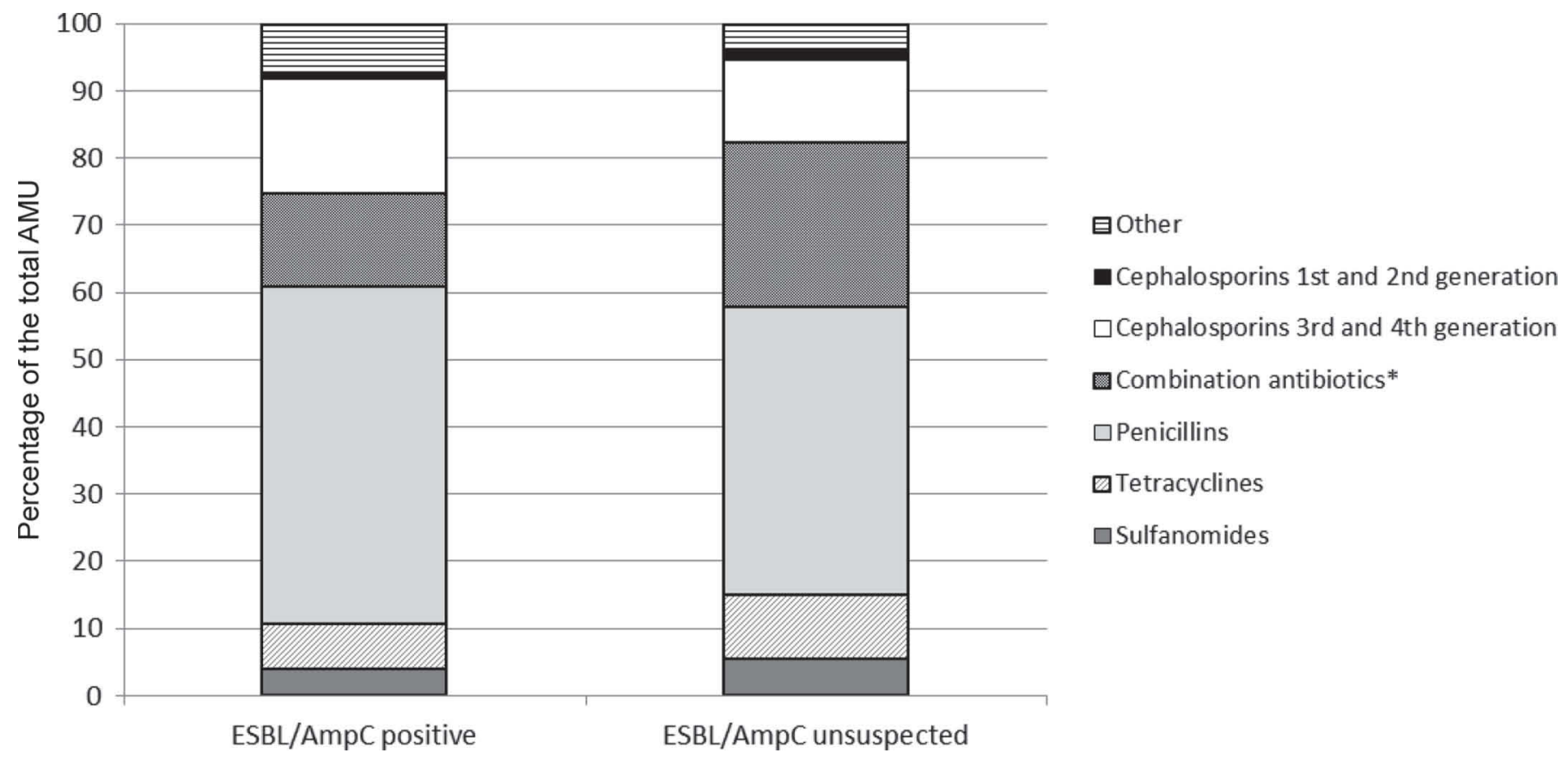

Figure 1. The percentage per type of antimicrobials that were used relative to the total antimicrobial use for 87 organic Dutch dairy herds that either tested positive for extended-spectrum $\beta$-lactamase and AmpC-producing Escherichia coli (ESBL/AmpC) or were unsuspected based on slurry samples in 2011. ${ }^{*}$ Combinations of antibiotics include mostly trimetrophin and sulfanomides and to lesser extent combination antibiotics with among others penicillin. AMU = antimicrobial use.

The ESBL/AmpC were isolated from slurry samples collected at 12 of the 90 herds $(13 \% ; 95 \% \mathrm{CI}=7-22 \%)$. Ten of these E. coli isolates were ESBL-producing and 2 were AmpC-producing. Nineteen of the slurry samples originated from scraper samples and 71 slurry samples were pooled manure samples from 5 places of the slatted floors. From the scraper samples, 4 tested positive $(21 \% ; 95 \% \mathrm{CI}=6-46 \%)$, the remaining 8 ESBL/AmpC-positive samples originated from the pool sampling $(11 \% ; 95 \% \mathrm{CI}=5-21 \%)$. The difference in prevalence of ESBL/AmpC between both sampling methods was not significant $(P=0.30)$.

\section{Use of Antimicrobials and the Association with ESBL/AmpC Herd Status}

From the 90 study herds, 3 farmers did not give consent to use their records on AMU. Hence, records on 479 deliveries of antimicrobials to 87 organic herds could be used for analysis. Six supplies (originating from 6 different herds) were removed from the data because it was unclear whether the antimicrobials were used for cattle or other species. Eventually, 473 supplies of antimicrobials to 87 dairy herds were included in the analysis.

The median $\mathrm{DDDA}_{\mathrm{F}}$ in organic dairy herds was 0.53 (mean 0.77 ) and varied between 0.00 and 4.83. Seven of the 87 herds (8\%; $95 \%$ CI: $3-16 \%)$ did not use any antimicrobials at all in 2011. The total $\mathrm{DDDA}_{\mathrm{F}}$ did not differ significantly between ESBL/AmpC-positive or -unsuspected organic dairy herds (both 0.53). The $\mathrm{DDDA}_{\mathrm{F}}$ of the application methods, $\mathrm{DDDA}_{\mathrm{F} \text {,oral, }}$ $\mathrm{DDDA}_{\mathrm{F}, \mathrm{dry}}$, and $\mathrm{DDDA}_{\mathrm{F} \text {,mast }}$, was not significantly different between ESBL/AmpC-positive and -unsuspected organic dairy herds either.

In Figure 1, the proportional use of the different groups of antimicrobials is shown. Several differences in the proportional use of groups of antimicrobials in ESBL/AmpC-positive and -unsuspected organic dairy herds were visible. For example, the proportional use of third- and fourth-generation cephalosporins was $17 \%$ in ESBL/AmpC-positive herds and $12 \%$ in unsuspected herds. However, differences between the proportions were not significant between ESBL/AmpC-positive and -unsuspected organic dairy herds.

In ESBL/AmpC-positive organic dairy herds, the median $\mathrm{DDDA}_{\mathrm{F}}$ of third- and fourth-generation cephalosporins was 0.02 (mean 0.14), compared with 0.00 (mean 0.09) in organic herds with an unsuspected ESBL/AmpC herd status. Although the DDDAF of third- and fourth-generation cephalosporins was higher in ESBL/AmpC-positive organic herds, this difference was not significant as compared with ESBL/AmpCunsuspected organic herds. Also, no significant asso- 
ciation was found between ESBL/AmpC herd status and the use of (amino)penicillins or in any of the other antimicrobial groups.

\section{Risk Factors for the Presence of ESBL/AmpC}

All 90 dairy herds that were included in the study completed the questionnaire. Based on the results of the univariable analyses, 17 variables were associated $(P<0.25$; Wald test) with being ESBL/AmpC-positive and were evaluated in the multivariable analysis (Table $2)$.

The final multivariable model contained 3 variables and explained $13 \%$ (pseudo $\mathrm{R}^{2}=0.13$ ) of the herd-level probability to be ESBL/AmpC-positive. Organic dairy herds of which the barn was located within a $2-\mathrm{km}$ radius to a pig farm had a 7.5 times higher odds to have an ESBL/AmpC-positive status compared with herds that were not located near a pig farm (Table 3). Applying parental treatment for part of the clini-

Table 2. Descriptive results of the univariable logistic regression analyses of extended-spectrum $\beta$-lactamase and AmpC-producing Escherichia coli $(\mathrm{ESBL} / \mathrm{AmpC})$ herd status and associated management factors $(P<0.25)$ in 90 Dutch organic dairy farms in 2011

\begin{tabular}{|c|c|c|c|}
\hline Parameter [no. (\%), unless noted otherwise] & $\begin{array}{l}\text { ESBL/AmpC- } \\
\text { unsuspected herds } \\
(\mathrm{n}=78)\end{array}$ & $\begin{array}{l}\text { ESBL/AmpC- } \\
\text { positive herds } \\
\quad(\mathrm{n}=12)\end{array}$ & $\begin{array}{c}P \text {-value } \\
\text { (Wald test) }\end{array}$ \\
\hline \multicolumn{4}{|l|}{ Presence of horses on the farm } \\
\hline No & $49(63)$ & $11(92)$ & \multirow[t]{2}{*}{0.08} \\
\hline Yes & $29(37)$ & $1(8)$ & \\
\hline \multicolumn{4}{|l|}{ Presence of cats on the farm } \\
\hline No & $8(10)$ & $5(42)$ & \multirow[t]{2}{*}{$<0.01$} \\
\hline Yes & $70(90)$ & $7(58)$ & \\
\hline \multicolumn{4}{|l|}{ Are any pig farms located within a radius of $2 \mathrm{~km}$ of the barn? } \\
\hline No & $55(71)$ & $5(42)$ & \multirow[t]{2}{*}{0.06} \\
\hline Yes & $23(29)$ & $7(58)$ & \\
\hline \multicolumn{4}{|l|}{ Type of milk that is fed to the female calves after colostrum intake } \\
\hline Milk replacer & $6(8)$ & $4(33)$ & \multirow[t]{2}{*}{0.02} \\
\hline Whole milk & $72(92)$ & $8(67)$ & \\
\hline \multicolumn{4}{|l|}{ Type of milk that is fed to the male calves after colostrum intake } \\
\hline Milk replacer & $5(6)$ & $3(25)$ & \multirow[t]{2}{*}{0.05} \\
\hline Whole milk (including milk of high-SCC cows and cows treated with antimicrobials) & $73(94)$ & $9(75)$ & \\
\hline \multicolumn{4}{|l|}{ What type of housing is used for the milking cows? } \\
\hline Cubicles in combination with slatted floors & $46(59)$ & $6(50)$ & \multirow[t]{3}{*}{0.23} \\
\hline Cubicles in combination with a solid floor & $9(12)$ & $3(25)$ & \\
\hline Other & $23(29)$ & $3(25)$ & \\
\hline \multicolumn{4}{|l|}{$\begin{array}{l}\text { Are antimicrobials used for treatment of calves }(<1 \text { yr old }) \text { for diseases other than } \\
\text { respiratory syndromes or diarrhea, such as lameness? }\end{array}$} \\
\hline No & $40(51)$ & $3(25)$ & \multirow[t]{2}{*}{0.10} \\
\hline Yes & $38(49)$ & $9(75)$ & \\
\hline \multicolumn{4}{|l|}{ Which type of milking system is used? } \\
\hline Conventional & $66(85)$ & $8(67)$ & \multirow[t]{2}{*}{0.15} \\
\hline Automatic milking system & $12(15)$ & $4(33)$ & \\
\hline \multicolumn{4}{|l|}{ Are you sometimes applying parental treatment for clinical mastitis } \\
\hline No & $51(65)$ & $4(33)$ & \multirow[t]{2}{*}{0.04} \\
\hline Yes & $27(35)$ & $8(67)$ & \\
\hline \multicolumn{4}{|l|}{ Last bulk tank milk SCC in 2011} \\
\hline Average $\times 10^{3}$ cells $/ \mathrm{mL}$ (range) & $210(70-389)$ & $237(113-320)$ & 0.21 \\
\hline \multicolumn{4}{|l|}{ Herd size $(>2$ yr old $)$} \\
\hline Average number of cows (range) & $62(10-185)$ & $78(29-186)$ & 0.22 \\
\hline \multicolumn{4}{|l|}{ Duration of antimicrobial treatment for lameness } \\
\hline Average number days (range) & $1.2(0-5)$ & $2.1(0-5)$ & 0.09 \\
\hline \multicolumn{4}{|l|}{$\begin{array}{l}\text { Percentage of cows ( }>2 \text { yr old) with antimicrobial treatment for other diseases } \\
\text { (besides mastitis and lameness) }\end{array}$} \\
\hline Average percentage (range) & $3.9(0-33)$ & $1.5(0-5)$ & 0.18 \\
\hline \multicolumn{4}{|l|}{$\begin{array}{l}\text { Percentage of calves ( }<1 \text { yr old) with antimicrobial treatment for other diseases } \\
\text { (besides lameness) }\end{array}$} \\
\hline Average percentage (range) & $6.0(0-50)$ & $12.9(0-50)$ & 0.10 \\
\hline \multicolumn{4}{|l|}{ Percentage of clinical mastitis cases that need parental treatment } \\
\hline Average percentage (range) & $17.9(0-100)$ & $35.8(0-100)$ & 0.07 \\
\hline \multicolumn{4}{|l|}{ Duration of parental treatment of clinical mastitis cows } \\
\hline Average number of days (range) & $1.2(0-8)$ & $2.1(0-5)$ & 0.09 \\
\hline Number of cows of which the udders are precleaned with one cloth & & & \\
\hline Average number of cows (range) & $5.6(1-37)$ & $2.1(1-4)$ & 0.15 \\
\hline
\end{tabular}


Table 3. Results of the multivariable logistic regression analysis modeling the probability of being extended-spectrum $\beta$-lactamase and AmpCproducing Escherichia coli (ESBL/AmpC)-positive for 90 organic Dutch dairy farms in 2011

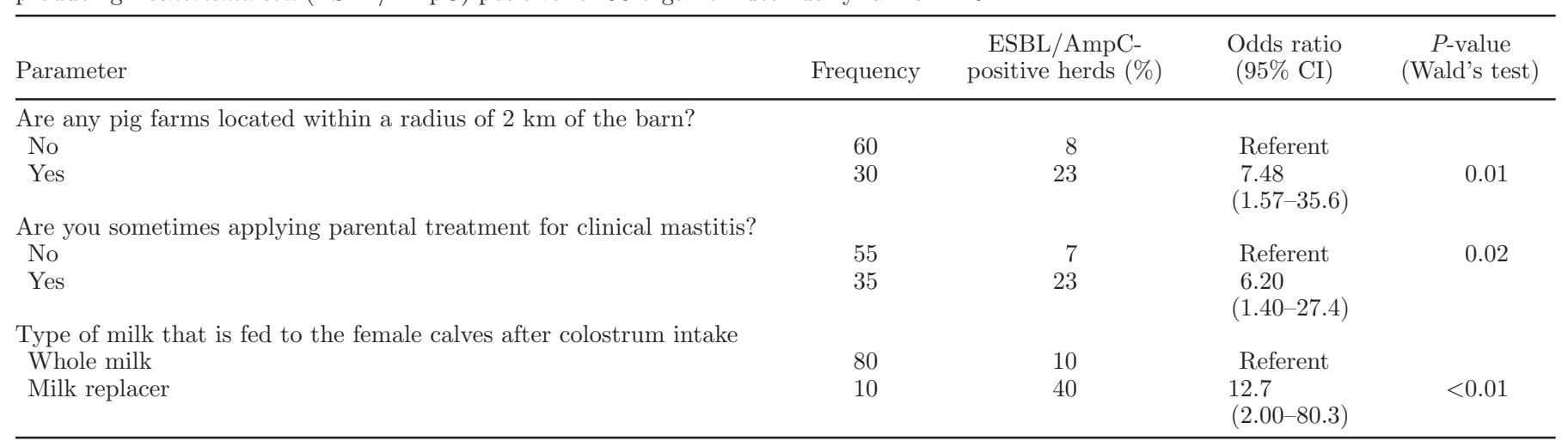

cal mastitis cases resulted in higher odds of having a positive ESBL/AmpC herd status as compared with never using parental treatment for treatment of clinical mastitis $(6.2 ; 95 \% \mathrm{CI}=1.4-27.4)$. Providing milk replacer to the calves after colostrum intake instead of whole milk was associated with a significantly higher odds $(12.7 ; 95 \%$ CI $=2.0-80.7)$ for being an ESBL/ AmpC-positive organic dairy herd (Table 3).

\section{DISCUSSION}

This study presented the prevalence of ESBL/AmpC in organic dairy herds and the association of the ESBL/ AmpC herd status with AMU and farm management. Based on the results, an ESBL/AmpC prevalence of $13 \%$ was estimated. In a previous study evaluating the ESBL/AmpC prevalence in conventional Dutch dairy herds using the same sampling method in the same time period, $41 \%$ of the herds tested positive (Gonggrijp et al., 2016). Other studies also found ESBL/AmpC herd prevalence in cattle herds that were higher compared with those found in the organic herds in our study, ranging between 25 and $86.7 \%$ (Mollenkopf et al., 2012; Snow et al., 2012; Schmid et al., 2013; Wu et al., 2013; Carmo et al., 2014). Although individual differences exist, conventional herds generally have a higher AMU than organic herds. Other differences are present between conventional and organic herds, such as herd size and milk production, but it may be suggested that the ESBL/AmpC status in dairy herds is associated with differences in AMU. Dolejska et al. (2011) found a large difference in ESBL/AmpC prevalence between cattle housed in a conventional and an organic dairy herd (39 and $<1 \%$, respectively) and suggested a possible association between ESBL/AmpC herd status and AMU. Nevertheless, in the study of Gonggrijp et al. (2016) the only significant effect of AMU on the ESBL/AmpC herd status was the use of third- and fourth-generation cephalosporins, not the total AMU. Tragesser et al. (2006) also found an association between the use of ceftiofur (third-generation cephalosporin) and reduced susceptibility of ceftiofur against E. coli on herd level. On a cow level (both lactating and nonlactating), Tragesser et al. (2006) could not confirm this association and Mollenkopf et al. (2012) could also not confirm the association between ceftiofur use and E. coli CTX-M/ CMY-2 positive isolates. In the current study, which was executed at herd level, we could not confirm the association between the use of third- and fourth-generation cephalosporins and ESBL/AmpC herd status in organic herds, which may be due to lack of power due to the low use of this type of antimicrobials in organic dairy herds. Although the ESBL/AmpC herd prevalence in the organic dairy herds in our study was low with $13 \%$, it was not negligible; thus, the use of third- and fourth-generation cephalosporins apparently is not the only factor associated with the ESBL/AmpC status in dairy herds.

Three factors were found to be significantly associated with the ESBL/AmpC herd status in organic dairy herds. To our knowledge, ours is the first study investigating factors relating to ESBL/AmpC status in organic dairy herds, and our findings could therefore not be compared with previous findings on organic dairy herds in literature. Organic dairy farmers that indicated that they sometimes applied parental treatment for clinical mastitis cases had higher odds to be ESBL/AmpC-positive. It might be that these farmers used higher amounts of third- and fourth-generation cephalosporins (cefoperazone or cefquinome) to treat clinical mastitis, which are known to be associated with a higher probability of ESBL/AmpC-positive E. coli (Tragesser et al., 2006; Snow et al., 2012; Gonggrijp et al., 2016). This finding, however, could not be confirmed in our study. Although ESBL/AmpC-positive herds used products containing third- and fourth-gen- 
eration cephalosporins more often than ESBL/AmpCunsuspected herds, this difference was not statistically significant.

Organic herds located in the proximity of pig farms were more likely to be ESBL/AmpC-positive. Whether the neighboring pig farms in our study were ESBL/ AmpC-positive and environmental contamination could have occurred was not evaluated. In earlier research, in $40 \%$ of the Dutch pig farms ESBL/AmpC-positive pigs were found (Hordijk et al. 2013b). In addition, a German study found that ESBL/AmpC could be present in dust samples of fattening pig farms (Hamscher et al., 2003). Blaak et al. (2015) found that ESBL-producing $E$. coli can spread through dust and pose a risk for transmission through the environment. As Gonggrijp et al. (2016) found that most ESBL genotypes in dairy herds also occur in isolates from pig farms, a relation between ESBL/AmpC in pig and dairy herds cannot be excluded.

Organic herds that supplied milk replacer to female calves instead of whole milk had higher odds for being ESBL/AmpC-positive. Only 10 organic herds fed milk replacer to female calves, of which 4 tested ESBL/ AmpC-positive. The reasoning for providing milk replacer instead of whole milk might be related to herd size, as herds that provided milk replacer were significantly larger compared with herds that fed whole milk (median 83 vs. 50 cows). Herd size was, however, also included in the questionnaire, but did not remain in the final model. In addition, organic dairy herds that fed milk replacer to their female calves appeared to have a significantly higher $\mathrm{DDDA}_{\mathrm{F}}$ in calves $(0-56 \mathrm{~d}$ of age) compared with farmers that fed whole milk to the calves (results not shown). Thus, the reasoning of finding feeding milk replacer as risk factor is probably multifactorial.

In our study, the total amount of $\mathrm{DDDA}_{\mathrm{F}}$, the amount per application method, and the AMU per specific group of antimicrobials were not significantly associated with the ESBL/AmpC herd status. Nevertheless, restriction of the total AMU remains important because AMU and the use of specific types of antimicrobials, such as penicillin, are known to enhance the emergence of other types of antimicrobial resistance, such as methicillin resistance in Staphylococcus aureus and vancomycin resistance in enterococci (Dahms et al., 2014).

In our study, 2 sampling methods were applied: sampling the scraper and, in herds without a scraper, sampling 5 different places on the slatted floor. Based on earlier work from Lombard et al. (2012) and Schmid et al. (2013), pooled slurry samples were expected to be sensitive enough to determine the ESBL/AmpC herd status. Nevertheless, as indicated by Gonggrijp et al.
(2016), some misclassification bias may have occurred in herds housing a small number of ESBL/AmpCpositive cattle, resulting in a slight underestimation of the ESBL/AmpC herd status. Our results showed no significant effect of sampling method on the ESBL/ AmpC herd status, and including sampling method in the risk factor analysis did not change the final model. Although we found no significant effect of sampling method, the ESBL/AmpC herd-level prevalence did seem higher in herds in which the scraper was sampled, which was in accordance with the results of Gonggrijp et al. (2016). Nevertheless, we believe that our results are valid because herds that tested negative in the slurry were either truly negative or likely had a low ESBL/AmpC prevalence.

In our study, we analyzed the association between ESBL/AmpC herd status and the management information obtained from the questionnaire separately from the information on the amount of (different types of) antimicrobials that were supplied by the veterinarian. We did not find a significant association between AMU and the ESBL/AmpC herd status in organic dairy herds. This might be caused by the fact that organic herds use antimicrobials sporadically, in combination with the fact that the ESBL/AmpC herd status was only evaluated at one point in time during the year of study. In dairy herds, AMU is, in general, not equally distributed throughout the year. Antimicrobials are applied for treatment of clinical disease, which occur throughout the year at different points in time in different herds. Although this might have slightly biased our results as related to the moment of sampling, we believe the influence was negligible because AMU in these herds was very low. Ideally, the study would be designed as cohort in which the ESBL/AmpC herd status would be evaluated during multiple sample moments. With such a study design, it would be possible to study the association between AMU and ESBL/ AmpC herd status in more detail.

In the study of Gonggrijp et al. (2016), ESBL/ AmpC-positive isolates were genotyped, revealing that the most frequently found ESBL genotypes originated from CTX-M group 1, 2, or 9 and sporadically they found the genotypes $b l a_{\mathrm{TEM}-52}$ and $b l a_{\mathrm{CMY}-2}$. Both the genotypes and their distribution were comparable to genotypes that were found in other European studies in cattle. The 12 positive isolates in our study were of the types CTX-M group 1 ( $\mathrm{n}=7$, CTX-M-1; $\mathrm{n}=1$, CTXM-15), CTX-M group $2(\mathrm{n}=1$, CTX-M-2), CTX-M group $9\left(\mathrm{n}=1\right.$, CTX-M-14), and $b l a_{\mathrm{CMY}-2}(\mathrm{n}=2)$, and were also not different from genotypic patterns found in European conventional dairy herds.

In a sensitivity analyses, we evaluated whether adding the information on the amount of antimicrobials 
that were supplied to the study herds would improve the final risk factor model. Adding this information did not result in a significant improvement of the model and it was decided not to include the information on AMU in the final risk factor model. The variance explained by the final model was only $13 \%$, which indicates other factors are likely associated with ESBL/AmpC herd status that were not included in our study.

\section{CONCLUSIONS}

The prevalence of ESBL/AmpC in Dutch organic dairy herds was $13 \%$ and appeared lower compared with conventional dairy herds. The total amount of AMU in organic dairy herds was very low and not associated with ESBL/AmpC herd status. No significant association was determined between the use of specific types of antimicrobials and the ESBL/AmpC herd status. A small number of management factors relating to AMU and possible environmental contamination were found to be associated with the ESBL/AmpC herd status. Apparently, ESBL/AmpC-producing E. coli are also present in herds with low AMU, indicating that other factors than AMU are also associated with ESBL/ AmpC herd status

\section{ACKNOWLEDGMENTS}

This study was financed by the Dutch Commodity board for Dairy (PZ, Zoetermeer, the Netherlands). We thank all farmers for their participation in this study.

\section{REFERENCES}

Akaike, H. 1974. A new look at the statistical model identification. IEEE Trans. Automat. Contr. 19:716-723.

Blaak, H., A. H. A. M. Van Hoek, R. A. Hamidjaja, R. Q. J. Van der Plaats, L. Kerkhof-de Heer, A. M. de Roda Huisman, and F. M. Schets. 2015. Distribution, numbers, and diversity of ESBL producing $E$. coli in the poultry farm environment. PLoS One 10:e0135402. https://doi.org/10.1371/journal.pone.0135402.

Carmo, L. P., L. R. Nielsen, P. M. da Costa, and L. Alban. 2014. Exposure assessment of extended-spectrum beta-lactamases/AmpC beta-lactamases-producing Escherichia coli in meat in Denmark. Infect. Ecol. Epidemiol. 4:22924. https://doi.org/10.3402/iee. $\mathrm{v} 4.22924$.

CLSI, 2011. Performance Standards for Antimicrobial Susceptibility Testing: Twenty-first Informational Supplement M100-21. Clinical and Laboratory Standards Institute, Wayne, PA.

Dahms, C., N. O. Hübner, F. Wilke, and A. Kramer. 2014. Minireview: Epidemiology and zoonotic potential of multiresistant bacteria and clostridium difficile in livestock and food. GMS Hyg. Infect. Control 9:Doc21. https://doi.org/10.3205/dgkh000241.

Dierikx, C. M., E. Van Duijkeren, A. H. Schoormans, A. Van EssenZandbergen, K. Veldman, A. Kant, X. W. Huijsdens, K. Van der Zwaluw, J. A. Wagenaar, and D. J. Mevius. 2012. Occurrence and characteristics of extended-spectrum- $\beta$-lactamase- and AmpCproducing clinical isolates derived from companion animals and horses. J. Antimicrob. Chemother. 67:1368-1374.
Dolejska, M., Z. Jurcickova, I. Literak, L. Pokludova, J. Bures, A. Hera, L. Kohoutova, J. Smola, and A. Cizek. 2011. IncN plasmids carrying bla CTX-M-1in Escherichia coli isolates on a dairy farm. Vet. Microbiol. 149:513-516.

Dorado-García, A., D. J. Mevius, J. J. H. Jacobs, I. M. Van Geijlswijk, J. W. Mouton, J. A. Wagenaar, and D. J. Heederik. 2016. Quantitative assessment of antimicrobial resistance in livestock during the course of a nationwide antimicrobial use reduction in the Netherlands. J. Antimicrob. Chemother. https://doi.org/10.1093/jac/ dkw308.

EC. 2007. Council Regulation (EC) No. 834/2007 of 28 June 2007 on organic production and labelling of organic products and repealing Regulation (EEC) No. 2092/91. Accessed Jan. 31, 2016. http:// www.wipo.int/wipolex/en/details.jsp?id $=7876$

EC. 2008. Commission Regulation (EC) No 889/2008 of 5 September 2008 laying down detailed rules for the implementation of Council Regulation (EC) No 834/2007 on organic production and labelling of organic products with regard to organic production, labelling and control. Accessed Jan. 31, 2016. http://eur-lex.europa.eu/ legal-content/nl/ALL/?uri=CELEX:32008R0889.

Gonggrijp, M. A., I. M. G. A. Santman-Berends, A. E. Heuvelink, J. J. Hage, G. J. Buter, G. Van Schaik, and T. J. G. M. Lam. 2016 Prevalence and risk factors of extended spectrum beta-lactamaseand AmpC-producing E. coli in dairy farms. J. Dairy Sci. 99:90019013. https://doi.org/10.3168/jds.2016-11134.

Hamscher, G., H. T. Pawelzick, S. Sczesny, H. Nau, and J. Hartung. 2003. Antimicrobials in dust originating from a pig-fattening farm: A new source of health hazard for farmers? Environ. Health Perspect. 111:1590-1594.

Hordijk, J., J. A. Wagenaar, and D. Mevius. 2013b. Preventing ESBLs in the Dutch livestock industry. Tijdschr. Diergeneeskd. 138:156159. (in Dutch).

Hordijk, J., J. A. Wagenaar, A. van de Giessen, C. Dierikx, A. van Essen-Zandbergen, K. Veldman, A. Kant, and D. Mevius. 2013a. Increasing prevalence and diversity of ESBL/AmpC-type $\beta$-lactamase genes in Escherichia coli isolated from veal calves from 1997 to 2010. J. Antimicrob. Chemother. 68:1970-1973.

Li, X. Z., M. Mehrotra, S. Ghimire, and L. Adewoye. 2007. $\beta$-lactam resistance and $\beta$-lactamases in bacteria of animal origin. Vet. Microbiol. 121:197-214.

Lombard, J. E., A. L. Beam, E. M. Nifong, C. P. Fossler, C. A. Kopral, D. A. Dargatz, B. A. Wagner, M. M. Erdman, and P. J. FedorkaCray. 2012. Comparison of individual, pooled, and composite fecal sampling methods for detection of Salmonella on U.S. dairy operations. J. Food Prot. 75:1562-1571.

MARAN. 2015. Monitoring of Antimicrobial Resistance and Antibiotic Usage in Animals in the Netherlands in 2014. Accessed September 2016. http://www.wur.nl/upload_mm/2/2/2/0ab4b3f5-1cf0-42e7a460-d67136870ae5_NethmapMaran2015.pdf.

Marshall, B. M., and S. B. Levy. 2011. Food animals and antimicrobials: impacts on human health. Clin. Microbiol. Rev. 24:718-733.

Meyer, E., P. Gastmeier, A. Kola, and F. Schwab. 2012. Pet animals and foreign travel are risk factors for colonisation with extendedspectrum-lactamase-producing Escherichia coli. Infection 40:685687.

Mollenkopf, D. F., M. F. Weeman, J. B. Daniels, M. J. Abley, J. L. Mathews, W. A. Gebreyes, and T. E. Wittum. 2012. Variable within- and between-herd diversity of CTX-M cephalosporinasebearing Escherichia coli isolates from dairy cattle. Appl. Environ. Microbiol. 78:4552-4560.

Reist, M., N. Geser, H. Hächler, S. Schärrer, and R. Stephan. 2013. ESBL-producing Enterobacteriaceae: Occurrence, risk factors for fecal carriage and strain traits in the Swiss slaughter cattle population younger than 2 years sampled at abattoir level. PLoS One 8:e71725. https://doi.org/10.1371/journal.pone.0071725.

Schmid, A., S. Hörmansdorfer, U. Messelhäusser, A. Käsbohrer, C. Sauter-Louis, and R. Mansefeld. 2013. Prevalence of extendedspectrum $\beta$-lactamase-producing Escherichia coli on Bavarian dairy and beef cattle farms. Appl. Environ. Microbiol. 79:30273032 . 
SDA. 2014. The use of fluorquinolons and 3rd and 4th generation cephalosporins in animal husbandry (in Dutch). Accessed Sep. 17, 2015. http://www.autoriteitdiergeneesmiddelen.nl/Userfiles/ pdf/rapportage-sda-ep-fluorochinolonen-en-3e-4e-generatiecefalosporinen-7-maart-2013.pdf.

Sharp, H., L. Valentin, Y. Fischer, B. Guerra, B. Appel, and A. Käsbohrer. 2014. Estimation of the transfer of ESBL-producing Escherichia coli to humans in Germany. Berl. Munch. Tierarztl. Wochenschr. 127:464-477.

Snow, L. C., R. G. Warner, T. Cheney, H. Wearing, M. Stokes, K. Harris, C. J. Teale, and N. G. Coldham. 2012. Risk factors associated with extended spectrum beta-lactamase Escherichia coli (CTX-M) on dairy farms in North West England and North Wales. Prev. Vet. Med. 106:225-234.

StataCorp. 2014. Stata Software version 13. Stata Corporation, College Station, TX.

Stefani, S., I. Giovanelli, I. Anacarso, C. Condò, P. Messi, S. de Niederhäusern, M. Bondi, R. Iseppi, and C. Sabia. 2014. Prevalence and characterization of extended-spectrum $\beta$-lactamase-producing Enterobacteriaceae in food-producing animals in northern Italy. New Microbiol. 37:551-555.

Teale, C. J., L. Barker, A. P. Foster, E. Liebana, M. Batchelor, D. M. Livermore, and E. J. Threlfall. 2005. Extended-spectrum betalactamase detected in $E$. coli recovered from calves in Wales. Vet. Rec. 156:186-187.

Thrusfield, M., C. Ortega, I. de Blas, J. P. Noordhuizen, and K. Frankena. 2001. WIN EPISCOPE 2.0: Improved epidemiological software for veterinary medicine. Vet. Rec. 148:567-572.
Tragesser, L. A., T. E. Wittum, J. A. Funk, P. L. Winokur, and P. J. Rajala-Schultz. 2006. Association between ceftiofur use and isolation of Escherichia coli with reduced susceptibility to ceftriaxone from fecal samples of dairy cows. Am. J. Vet. Res. 67:1696-1700.

Valentin, L., H. Sharp, K. Hille, U. Seibt, J. Fischer, Y. Pfeifer, G. B. Michael, S. Nickel, J. Schmiedel, L. Falgenhauer, A. Friese, R. Bauerfeind, U. Roesler, C. Imirzalioglu, T. Chakraborty, R. Helmuth, G. Valenza, G. Werner, S. Schwarz, B. Guerra, B. Appel, L. Kreienbrock, and A. Käsbohrer. 2014. Subgrouping of ESBLproducing Escherichia coli from animal and human sources: An approach to quantify the distribution of ESBL types between different reservoirs. Int. J. Med. Microbiol. 7:805-816. https://doi. org/10.1016/j.ijmm.2014.07.015.

von Wintersdorff, C. J., J. Penders, E. E. Stobberingh, A. M. Lashof, C. J. Hoebe, P. H. Savelk-oul, and P. F. Wolffs. 2014. High rates of antimicrobial drug resistance geneacquisition after international travel, the Netherlands. Emerg. Infect. Dis. 20:649-657.

Wu, G.. M. J. Day, M. T. Mafura, J. Nunez-Garcia, J. J. Fenner, M. Sharma, A. van Essen-Zandbergen, I. Rodríguez, C. Dierikx, K. Kadlec, A. K. Schink, M. Chattaway, J. Wain, R. Helmuth, B. Guerra, S. Schwarz, J. Threlfall, M. J. Woodward, N. Woodford, N. Coldham, and D. Mevius. 2013. Comparative analysis of ESBL-positive Escherichia coli isolates from animals and humans from the UK, The Netherlands and Germany. PLoS One 8:e75392. https://doi.org/10.1371/journal.pone.0075392. 